\title{
Effect of cyclic chain architecture on properties of dilute solutions of polyethylene from molecular dynamics simulations
}

\author{
Seung Soon Jang, Tahir Çağin, and William A. Goddard III \\ Materials and Process Simulation Center, Beckman Institute, California Institute of Technology, \\ Pasadena, California 91125
}

(Received 6 February 2003; accepted 16 April 2003)

\begin{abstract}
We have used molecular dynamics methods to investigate the effects of cyclic chain architecture on the properties of dilute solutions. In order to include solvent effects in estimating these properties, we use a van der Waals scaling factor determined for each solvent by matching to the theta condition. We predict that the theta temperature $(\theta)$ of cyclic PE (c-PE) is $\sim 10 \%$ lower than for the linear case (1-PE). This can be compared to the experimental results for polystyrene (PS), where $\theta$ for cyclic PS is $2 \%$ lower. For conditions corresponding to $n$-pentane solvent, we predict that $\left\langle R_{g}^{2}\right\rangle_{\text {cyclic }} /\left\langle R_{g}^{2}\right\rangle_{\text {linear }}$ is 0.59 for all temperatures above $350 \mathrm{~K}$. The deviation from the ratio of $0.50-0.53$ expected from analytic theory is due to the competition between chain stiffness and excluded volume effects. To calculate the intrinsic viscosity of c-PE and l-PE we extended the Bloomfield-Zimm type theory to include chain stiffness corrections. We find that for the theta temperature, the ratio of viscosities for c-PE and 1-PE is 0.71 , which is $7 \%$ higher than the value of 0.66 from the freely jointed chain model. This difference is caused by the larger value of $\left\langle R_{g}^{2}\right\rangle_{\text {cyclic }} /\left\langle R_{g}^{2}\right\rangle_{\text {linear }}$ from the simulations. (C) 2003 American Institute of Physics.

[DOI: $10.1063 / 1.1580802]$
\end{abstract}

\section{INTRODUCTION}

The recent breakthrough in synthesizing monodisperse cyclic ethylene by the Grubbs group ${ }^{1}$ stimulated us to investigate the effects of cyclic chain architecture on the properties of dilute solutions. Since the properties of dilute polymer solutions depend upon the spatial distribution of monomeric units and on the number of monomeric unit in a chain, ${ }^{2-7}$ the chain architecture (e.g., linear, star, comb, or ring) will affect the hydrodynamic properties even with the same number of monomeric units. The unique topology of having no chain ends make cyclic chains of particular interest. ${ }^{8-10}$ Thus, the observation that viral DNA can be circular ${ }^{1-18}$ stimulated theoretical studies to predict the difference between cyclic chain and linear counterparts to elucidate the effect of cyclic architecture. ${ }^{19-45}$ The synthesis of cyclic polymers with narrow molecular weight distributions ${ }^{46-58}$ now makes it feasible to verify such theoretical predictions.

Kramers ${ }^{8,9}$ estimated that the viscosity ratio between a cyclic polymer and its linear counterpart is 0.5 based on the observation that the dimension of a cyclic chain should be one half of that of linear polymer. A similar result was derived independently by Zimm and Stockmayer. ${ }^{10}$ Such considerations ignore both the hydrodynamic interactions and excluded volume effects. Later Zimm ${ }^{59}$ extended the Rouse model $^{60}$ by introducing hydrodynamic interactions between chain segments in a viscous medium for linear chains, and then Bloomfield and $\mathrm{Zimm}^{20}$ and Fukatsu and Kurata ${ }^{21}$ independently included the effects of hydrodynamic interactions for cyclic chains in dilute solution while the excluded volume effect ${ }^{61-64}$ was included by assuming the Kirkwood-

a) Author to whom all correspondence should be addressed.
Riseman approximation. ${ }^{65}$ The result for the theta condition was that $[\eta]_{c} /[\eta]_{l}=0.662$ (Bloomfield and Zimm) and 0.645 (Fukatsu and Kurata), where $[\eta]_{c}$ and $[\eta]_{l}$ are the intrinsic viscosity of the cyclic and linear polymers, respectively. Although in good agreement with each other, these two theoretical approaches employed conflicting assumptions to predict the excluded volume effect, resulting in opposite predictions for the radius of gyration ratio $\left(\left\langle R_{g}^{2}\right\rangle_{\text {cyclic }} /\left\langle R_{g}^{2}\right\rangle_{\text {linear }}\right.$ where $\left\langle R_{g}^{2}\right\rangle_{\text {cyclic }}$ and $\left\langle R_{g}^{2}\right\rangle_{\text {linear }}$ are the radius of gyration for cyclic and linear chain, respectively). Fukatsu and Kurata found that the $R_{g}$ ratio increases with increasing excluded volume effect, whereas Bloomfield and Zimm found the opposite trend. Consequently, the predicted $[\eta]_{c} /[\eta]_{l}$ by Bloomfield and Zimm is decreased faster than that by Fukatsu and Kurata. Later, Yu and Fujita ${ }^{4}$ improved the formula suggested by Bloomfield and Zimm, leading to the same trend as Fukatsu and Kurata. On the other hand, using excluded volume theory based on perturbation theory, ${ }^{66}$ Pyun and Fixman ${ }^{67,68}$ calculated the intrinsic viscosity of linear chain without the Kirkwood-Riseman approximation and found a 5\% smaller value of intrinsic viscosity. Later, Fixman pointed out that, in the KirkwoodRiseman approximation, a polymer chain would maintain its instantaneous confor mation during rotation or translation and hence this formalism neglects thermal fluctuation of the chain conformation. ${ }^{69}$ On this basis, Imai predicted the dependence of intrinsic viscosity ratio on solvent power, reaching qualitative result similar to the Bloomfield-Zimm type theory. They pointed out that the Bloomfield-Zimm type theory overestimates chain stiffness effects. ${ }^{23}$

Although, the predictions from these analytic theories are qualitatively correct at the theta condition, they cannot be 
TABLE I. Force field parameters used for coarse-grained cyclic and linear polyethylene. The functional forms are given in Eq. (1), ${ }^{\mathrm{a}, \mathrm{b}}$

\begin{tabular}{|c|c|c|c|c|c|c|c|}
\hline \multirow[t]{2}{*}{$E^{\mathrm{vdW}}$} & $\mathrm{CH}_{2}$ & $R_{0}{ }^{\mathrm{c}}$ & 4.4113 & $D_{0}{ }^{\mathrm{d}}$ & 0.09339 & & \\
\hline & $\mathrm{CH}_{3}$ & $R_{0}{ }^{\mathrm{c}}$ & 4.4113 & $D_{0}{ }^{\mathrm{d}}$ & 0.22650 & & \\
\hline \multirow{2}{*}{$E^{\text {bond }}$} & $\mathrm{CH}_{2}-\mathrm{CH}_{2}$ & $R_{0}{ }^{\mathrm{c}}$ & 1.54 & $K_{b}{ }^{\mathrm{e}}$ & 520 & & \\
\hline & $\mathrm{CH}_{2}-\mathrm{CH}_{3}$ & $R_{0}{ }^{\mathrm{c}}$ & 1.54 & $K_{b}{ }^{\mathrm{e}}$ & 520 & & \\
\hline \multirow[t]{2}{*}{$E^{\text {angle }}$} & $\mathrm{CH}_{2}-\mathrm{CH}_{2}-\mathrm{CH}_{2}$ & $\theta_{0}{ }^{\mathrm{f}}$ & 114 & $K_{\theta}{ }^{\mathrm{g}}$ & 124.19 & & \\
\hline & $\mathrm{CH}_{2}-\mathrm{CH}_{2}-\mathrm{CH}_{3}$ & $\theta_{0}{ }^{\mathrm{f}}$ & 114 & $K_{\theta}{ }^{\mathrm{g}}$ & 124.19 & & \\
\hline$E^{\text {torsion }}$ & $\begin{array}{l}\mathrm{CH}_{2}-\mathrm{CH}_{2}-\mathrm{CH}_{2}-\mathrm{CH}_{2} \\
\mathrm{CH}_{2}-\mathrm{CH}_{2}-\mathrm{CH}_{2}-\mathrm{CH}_{3}\end{array}$ & $\begin{array}{l}V_{1}^{\mathrm{d}}\left(d_{1}\right) \\
V_{1}^{\mathrm{d}}\left(d_{1}\right)\end{array}$ & $\begin{array}{l}1.4109(-1) \\
1.4109(-1)\end{array}$ & $\begin{array}{l}V_{2}^{\mathrm{d}}\left(d_{2}\right) \\
V_{2}^{\mathrm{d}}\left(d_{2}\right)\end{array}$ & $\begin{array}{l}-0.271(1) \\
-0.271(1)\end{array}$ & $\begin{array}{l}V_{3}^{\mathrm{d}}\left(d_{3}\right) \\
V_{3}^{\mathrm{d}}\left(d_{3}\right)\end{array}$ & $\begin{array}{l}2.787(-1) \\
2.787(-1)\end{array}$ \\
\hline
\end{tabular}

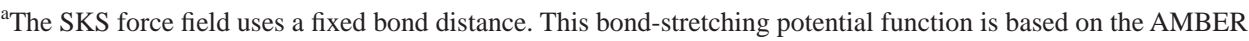
force field (Refs. 97 and 98).

${ }^{\mathrm{b}}$ The torsion potential of the SKS force field was based on the OPLS (optimized potentials for liquid simulation) force field (Ref. 99).

c In Å. SKS use $E^{\mathrm{vdW}}(R)=4 \epsilon\left\{(\sigma / R)^{12}-(\sigma / R)^{6}\right\}$. Thus $R_{0}=\sqrt[6]{2} \sigma$.

${ }^{\mathrm{d}}$ In $\mathrm{kcal} / \mathrm{mol} . D_{0}(\mathrm{kcal} / \mathrm{mol})=k \epsilon(\mathrm{SKS})$ where $k$ is the Boltzmann constant.

${ }^{\mathrm{e}}$ In $\mathrm{kcal} / \mathrm{mol} / \AA^{2}$.

In degrees.

${ }^{\mathrm{g}}$ In $\mathrm{kcal} / \mathrm{mol} / \mathrm{rad}^{2}$

compared directly to experimental observations ${ }^{46-58}$ in various solvents. Since these theories are derived assuming the simple freely jointed chain model, they cannot treat chain stiffness, which arises from local interactions between nearby neighboring segments. ${ }^{3,4,20,21}$ Consequently, we developed a theory to include the role of chain stiffness (which depends on the specific polymer) in the presence of excluded volume effects.

In this paper we focus on the effect of cyclic chain architecture with unknotted topology on dilute solution properties of polyethylene in various solvents by combining molecular dynamics (MD) simulations with the BloomfieldZimm type analytic theory. ${ }^{4,20}$

\section{MODEL AND SIMULATION METHOD}

\section{A. Force field}

The methylene $\left(\mathrm{CH}_{2}\right)$ and methyl $\left(\mathrm{CH}_{3}\right)$ groups in polyethylene were treated as united atoms, that is, each $\mathrm{CH}_{2}$ or $\mathrm{CH}_{3}$ unit was treated as a single neutral pseudoatom centered at the $\mathrm{C}$. The force field for this coarse-grained polyethylene model uses valence terms of the form

$$
\begin{aligned}
& E^{\text {bond }}(R)^{a}=\frac{1}{2} K_{R}\left(R-R_{0}\right)^{2}, \\
& E^{\text {angle }}(\theta)=\frac{1}{2} K_{\theta}\left(\theta-\theta_{0}\right)^{2}, \\
& E^{\text {torsion }}(\phi)^{b}=\sum_{n} \frac{1}{2} V_{n}\left[1-d_{n} \cos (n \phi)\right],
\end{aligned}
$$

where the parameters are summarized in Table I. It is based on the Siepmann-Karanorni-Smit force field ${ }^{70-72}$ developed to describe thermodynamic properties of $n$-alkanes. The van der Waals (vdW) interactions are described with LennardJones 12-6 potentials,

$$
E^{\mathrm{vdW}}(R)=D_{0}\left\{\left(\frac{R_{0}}{R}\right)^{12}-2\left(\frac{R_{0}}{R}\right)^{6}\right\}
$$

allowing different parameters for $\mathrm{CH}_{2}$ and $\mathrm{CH}_{3}$ groups. The off-diagonal vdW interactions are defined by the geometric mean combination rule: $R_{0}^{A B}=\sqrt{R_{0}^{A} R_{0}^{B}}$ and $D_{0}^{A B}=\sqrt{D_{0}^{A} D_{0}^{B}}$.
The nonbonded interactions between 1-2,1-3, and 1-4 neighbors were excluded. This force field was tested extensively by Smit and co-workers, ${ }^{70-73}$ for $n$-alkanes ranging from pentane $\left(\mathrm{C}_{5}\right)$ through octatetracontane $\left(\mathrm{C}_{48}\right)$ and is successful in describing both vapor-liquid phase equilibria and surface tension.

To include solvent effects without the enormous cost of explicitly treating huge numbers of solvent molecules in MD simulations, we decreased the strength $D_{0}$ of the nonbonded interactions. The idea is that

in a poor solvent (e.g., vacuum) the chain has a tendency to fold into a globule at lower temperatures; this folding into a globule is opposed by exclusion effects (vdW) and chain stiffness,

(ii) a good solvent will tend to dissolve the components of the chain so that the van der Waals interactions experienced by each atom arises mostly from the solvent, leading to a very open polymer chain topology.

To mimic such effects with an implicit model, we screen the total van der Waals interaction as in the following:

$$
E_{\mathrm{effective}}^{\mathrm{vdW}}(R)=f D_{0}\left\{\left(\frac{R_{0}}{R}\right)^{12}-2\left(\frac{R_{0}}{R}\right)^{6}\right\} .
$$

The solvation scale factor $f$ in Eq. (2) changes only the energy well depth without changing van der Waals radius. That is, we assume that positioning solvent molecules between polymer segments affects both the repulsive interaction and the attractive interaction to the same extent. Thus, the source of screening of van der Waals interaction is just the solvent molecules occupying the space between polymer segments. Here solvents with different screening efficiency or different solvent power will lead to a different scaling factor, $f$. This is similar to using a dielectric constant to mimic the screening of the charge-charge interactions by a solvent.

Previously, Sariban et al. ${ }^{74,75}$ and Ryckaert and his co-workers ${ }^{76}$ studied properties of polyethylene at the theta condition by introducing a reduced dispersion parameter, $\beta$, in the Lennard-Jones potential: 


$$
E^{\mathrm{vdW}}(R)=4 \epsilon\left\{\left(\frac{\sigma}{R}\right)^{12}-\beta\left(\frac{\sigma}{R}\right)^{6}\right\},
$$

where $\beta$ is between 0.0 and 1.0. Here the theta condition was found by adjusting the value of $\beta$ at a given value of $\epsilon$. Both groups reported a value for $\beta$ around 0.5 at $400 \mathrm{~K}$, but the physical meaning of this ad hoc parameter, $\beta$, has not been thoroughly discussed. Equation (3) leads to a vdW radius of $R_{0}=(2 / \beta)^{1 / 6} \sigma$ which increases with decreasing $\beta$ while the vdW energy of $D_{0}=-\epsilon \beta^{2}$ becomes weaker with decreasing $\beta$. Thus $\beta$ balances the attractive and repulsive interactions due to the solvent, allowing the chain expansion resulting from excluded volume effects to be compensated.

\section{B. Molecular dynamics}

Our molecular dynamics studies used the Nose-Hoover thermostat ${ }^{77-80}$ to maintain the temperature. The van der Waals interactions were calculated for all pairs of atoms without a finite cutoff distance.

We considered cyclic and linear polyethylene for chains with 200, 300, 400, and 600 atoms using CERIUS2 (from Accelyrs Inc. ${ }^{81}$ and MPSIM. ${ }^{82-85}$ To prepare the cyclic chains, we first built an ordinary linear chain and then brought the two chain ends gradually closer and closer by applying successive decreased distance constraints and minimizing the energy. After the two chain ends were close enough to make a covalent bond, we formed a bond between them. Before data collection, all the chains were equilibrated using $2 \mathrm{~ns} N V T$ MD simulation at each temperature. The van der Waals repulsion between various parts of the ring causes the initial unknotted topology to be retained throughout the simulations, i.e., bond crossing is not possible. Figure 1 shows some typical results. We generally carried out the MD simulations for $20 \mathrm{~ns}$ at various temperatures with three scaling factors: $f=0.25,0.50$, and 1.00 .

\section{DIMENSIONS OF LINEAR AND CYCLIC CHAINS IN SOLUTION}

\section{A. The Flory exponents}

The mean square radius of gyration of cyclic and linear polyethylene was calculated from the trajectories generated during MD simulations at various temperatures, by

$$
\left\langle R_{g}^{2}\right\rangle=\left\langle\frac{1}{N} \sum_{i=1}^{N}\left(\mathbf{r}_{i}-\mathbf{r}_{\mathrm{c} . \mathrm{m} .}\right)^{2}\right\rangle,
$$

where $\mathbf{r}_{i}$ denotes the position vector of each united atom $\left(\mathrm{CH}_{2}\right.$ or $\left.\mathrm{CH}_{3}\right)$ and $\mathbf{r}_{\text {c.m. }}$. denotes the position vector of the center-of-mass for the chain. Kramer ${ }^{8,9}$ and Zimm and Stockmayer ${ }^{10}$ suggested that the radius of gyration of cyclic and linear freely jointed chain has the following relationship with the number of bonds in a chain:

$$
\begin{array}{ll}
\left\langle R_{g}^{2}\right\rangle_{\text {cyclic }}=\frac{1}{12}(N-1) b^{2} & \text { for cyclic chain, } \\
\left\langle R_{g}^{2}\right\rangle_{\text {linear }}=\frac{1}{6}(N-1) b^{2} & \text { for linear chain, }
\end{array}
$$

where $b$ is the bond length. These relationships are valid only at the theta condition where the effects of excluded volume
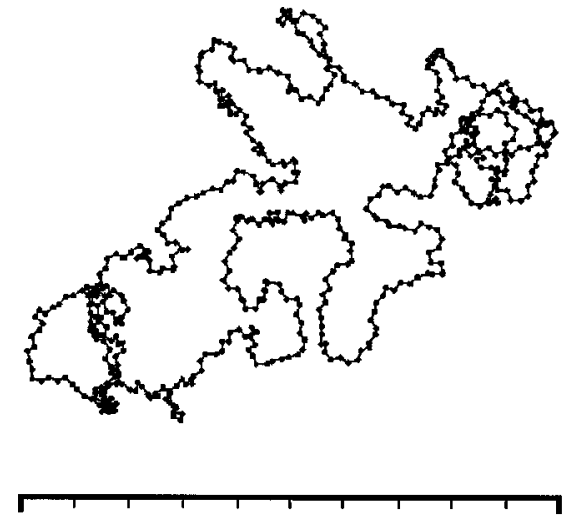

$10 \mathrm{~nm}$

(a) Cyclic polyethylene with 600 carbons

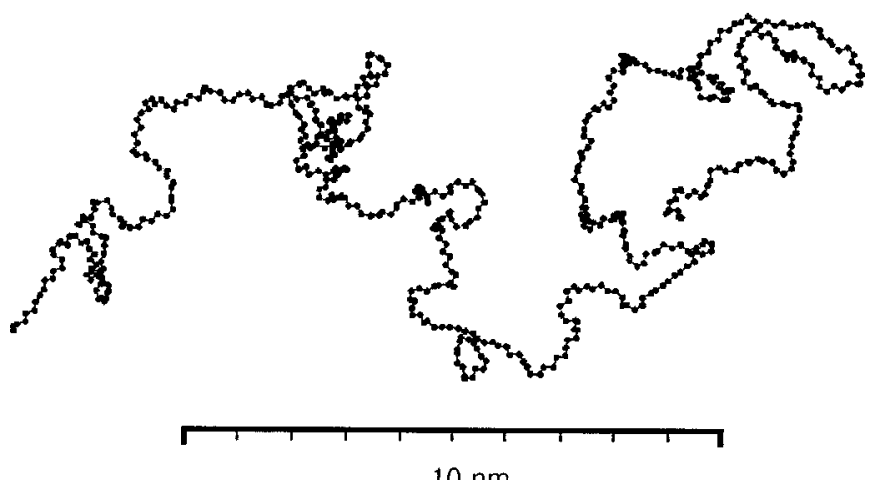

(b) Linear polyethylene with 600 carbons

FIG. 1. Typical structures arising in the MD simulations of linear and cyclic polyethylene. This is for $N=600$ at $T=600 \mathrm{~K}$ for solvent scaling factor of $f=0.25$ (appropriate for $N=600$ ).

are compensated by the solvent. In order to study the chain dimensions for various solvents, Eqs. (5a) and (5b) have been extended as follows: $:^{4,20}$

$$
\begin{aligned}
\left\langle R_{g}^{2}\right\rangle_{\text {cyclic }}= & \frac{1}{2\left(6+5 \zeta+\zeta^{2}\right)}\left(1+\frac{\zeta}{3+2 \zeta}\right)(N-1)^{2 \nu} b^{2} \\
& \text { for cyclic chain, } \\
\left\langle R_{g}^{2}\right\rangle_{\text {linear }}= & \frac{1}{\left(6+5 \zeta+\zeta^{2}\right)}(N-1)^{2 v} b^{2} \quad \text { for linear chain, }
\end{aligned}
$$

where $\zeta$, the excluded volume parameter, is defined as $\zeta$ $=2 \nu-1$, and $\nu$ is the Flory exponent. It should be noted that Eqs. (6a) and (6b) express asymptotic relationship of chain dimension with molecular weight (or chain length) which are valid at large $N$. So far, the Flory exponent, $\nu$ has been extensively studied through various theoretical frameworks such as the perturbation theory, the exact enumeration, the mean-field theory, and the renormalization group theory and all these theoretical works converge to the common feature in which it is governed by the excluded-volume-effect and has the value of 0.588 for a good solvent and 0.333 for a poor solvent, while the value is 0.5 for the theta condition. ${ }^{4-7}$ 

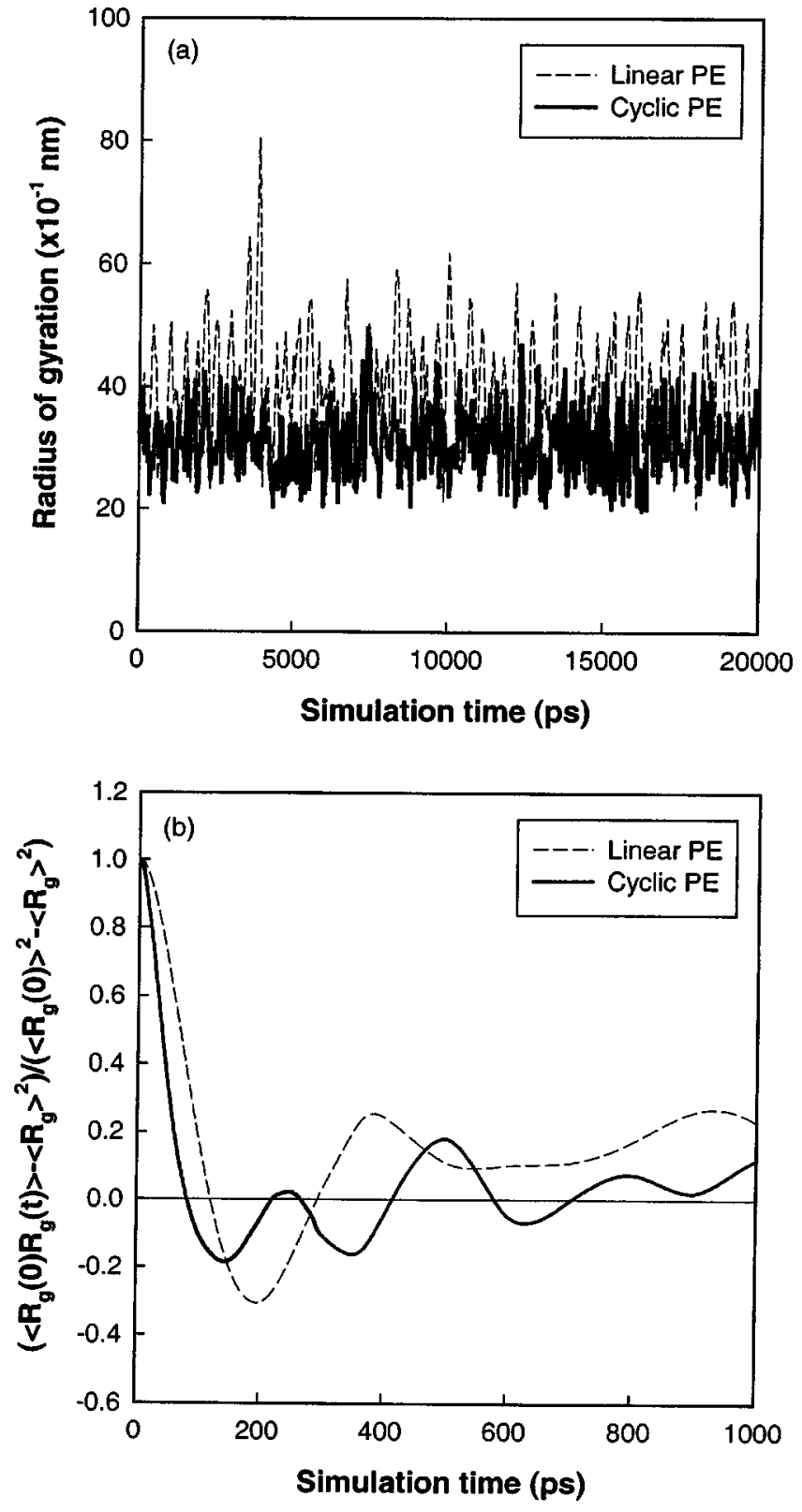

FIG. 2. (a) Time evolution of the radius of gyration for cyclic and linear PE for the case of $N=600$ carbons with solvent scaling ratio of $f=0.25$. Here $T=300 \mathrm{~K}$ for cyclic PE and $T=328 \mathrm{~K}$ for linear PE (their respective theta temperatures). (b) The autocorrelation functions for the trajectory in (a).

For various values of solvent shielding, $f$, we calculated $\left\langle R_{g}^{2}\right\rangle$ as a function of $N$ for a range of temperatures. Figure 2 shows typical results for $\left\langle R_{g}\right\rangle$ for cyclic and linear PE including its autocorrelation behavior. Through $20 \mathrm{~ns}$ of simulation time, it is obvious that chain samples conformational phase space sufficiently and that the autocorrelation decays rapidly (within $0.5 \mathrm{~ns}$ ). This indicates that $20 \mathrm{~ns}$ is sufficiently long to obtain converged results. Typical results are shown in Fig. 3 for a solvent scale factor of $f=0.25$ with temperatures from 200 to $600 \mathrm{~K}$ in $50 \mathrm{~K}$ increments (additionally, $328 \mathrm{~K}$ was simulated for linear polyethylene). Here, we see that

$$
\ln \left\langle R_{g}^{2}\right\rangle=\nu \ln (N-1)^{2}+\text { const },
$$
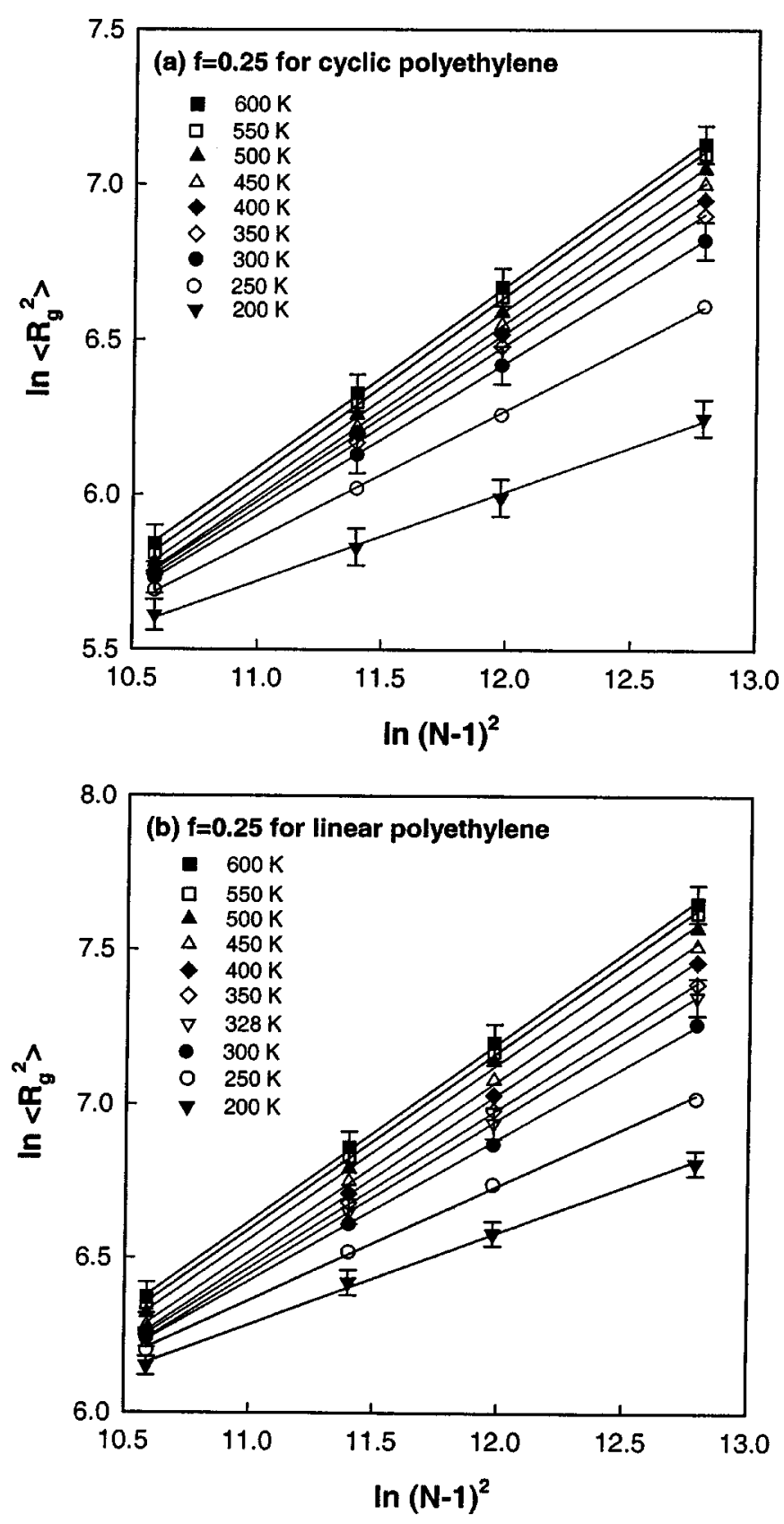

FIG. 3. Change of mean square radius of gyration with increasing length $(N)$ at various temperatures. The error bars correspond to the rms deviation calculated by considering the 20-ns-long segment as 10 uncorrelated $2 \mathrm{~ns}$ trajectories.

where the Flory exponent $\nu$ is evaluated from the slope of each linear regression curve, as shown in Fig. 4 and Table II. The value of $\nu$ decreases from $\sim 0.58$ for a good solvent to 0.3 for a poor solvent. These results are similar to the Monte Carlo studies using finite chain length ${ }^{86-88}$ which find the stepwise transition behavior smeared over a temperature range, as in our simulation. We expect that this smearing range will decrease as the chain length is increased.

\section{B. The theta temperature}

The point at which the intermolecular interactions with the solvent exactly compensate the excluded volume effects due to intramolecular interactions between polymer seg- 


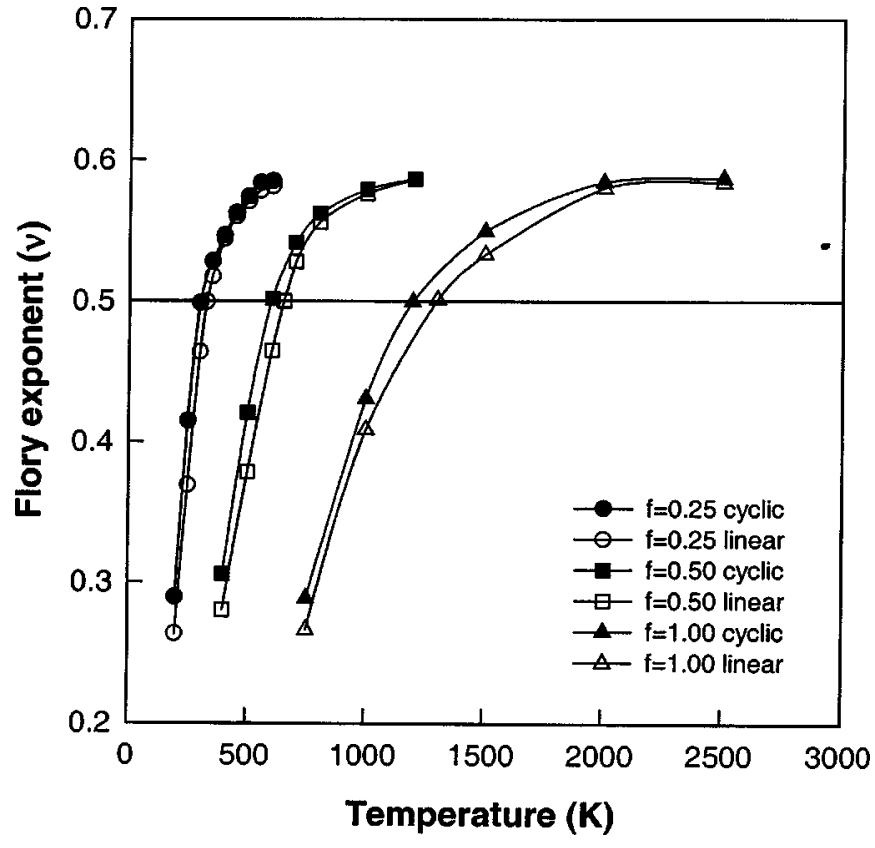

FIG. 4. Dependence of the Flory exponent on the solvent scaling factor $(f)$ for various temperatures. For cyclic chains the theta condition is found at $300 \mathrm{~K}$ for $f=0.25$, at $600 \mathrm{~K}$ for $f=0.50$, and at $1195 \mathrm{~K}$ for $f=1.00$. For linear chains the theta condition is found at $328 \mathrm{~K}$ for $f=0.25$, at $650 \mathrm{~K}$ for $f=0.50$, and at $1300 \mathrm{~K}$ for $f=1.00$.

ments leads to $\left\langle R_{g}^{2}\right\rangle$ proportional to $(N-1)$, a Gaussian coil. This is called the theta temperature $(\theta)$. From Eq. (7) we see that the Flory exponent is $\nu=0.5$ at $T=\theta$. The calculated values of $\theta$ for various $f$ are collected in Table III.

TABLE II. Flory exponents calculated for each solvent scale factor as a function of temperature.

\begin{tabular}{|c|c|c|c|}
\hline \multirow[b]{2}{*}{ Scale factor $(f)$} & \multirow[b]{2}{*}{ Temperature (K) } & \multicolumn{2}{|c|}{ Flory exponent $(\nu)$} \\
\hline & & Cyclic PE & Linear PE \\
\hline \multirow[t]{10}{*}{0.25} & 200 & 0.2893 & 0.2634 \\
\hline & 250 & 0.4150 & 0.3692 \\
\hline & 300 & 0.4986 & 0.4638 \\
\hline & 328 & $\cdots$ & 0.4995 \\
\hline & 350 & 0.5280 & 0.5174 \\
\hline & 400 & 0.5465 & 0.5439 \\
\hline & 450 & 0.5625 & 0.5598 \\
\hline & 500 & 0.5740 & 0.5705 \\
\hline & 550 & 0.5838 & 0.5782 \\
\hline & 600 & 0.5850 & 0.5812 \\
\hline \multirow[t]{8}{*}{0.50} & 400 & 0.3057 & 0.2803 \\
\hline & 500 & 0.4208 & 0.3785 \\
\hline & 600 & 0.5016 & 0.4648 \\
\hline & 650 & $\ldots$ & 0.5000 \\
\hline & 700 & 0.5415 & 0.5280 \\
\hline & 800 & 0.5622 & 0.5558 \\
\hline & 1000 & 0.5791 & 0.5760 \\
\hline & 1200 & 0.5865 & 0.5862 \\
\hline \multirow[t]{7}{*}{1.00} & 750 & 0.2886 & 0.2664 \\
\hline & 1000 & 0.4307 & 0.4087 \\
\hline & 1195 & 0.4996 & $\ldots$ \\
\hline & 1300 & $\cdots$ & 0.5010 \\
\hline & 1500 & 0.5494 & 0.5330 \\
\hline & 2000 & 0.5844 & 0.5801 \\
\hline & 2500 & 0.5870 & 0.5840 \\
\hline
\end{tabular}

TABLE III. Characteristic ratio of cyclic and linear polyethylene for simulations under various solvent scaling factors. See Eqs. (10) and (11).

\begin{tabular}{cccccc}
\hline \hline \multirow{2}{*}{$\begin{array}{c}\text { Solvent } \\
\text { scaling } \\
\text { factor }(f)\end{array}$} & \multicolumn{2}{c}{ Cyclic } & & \multicolumn{2}{c}{ Linear } \\
\cline { 2 - 3 } \cline { 5 - 6 } & $\begin{array}{c}\text { Theta } \\
\text { temperature }\end{array}$ & $C_{N, \text { cyclic }}$ & & $\begin{array}{c}\text { Theta } \\
\text { temperature }\end{array}$ & $C_{N, \text { linear }}$ \\
\hline 0.25 & 300 & $7.770 \pm 0.010$ & & 328 & $6.530 \pm 0.003$ \\
0.50 & 600 & $5.880 \pm 0.018$ & & 650 & $5.060 \pm 0.005$ \\
1.00 & 1195 & $5.480 \pm 0.023$ & & 1300 & $4.850 \pm 0.011$ \\
\hline \hline
\end{tabular}
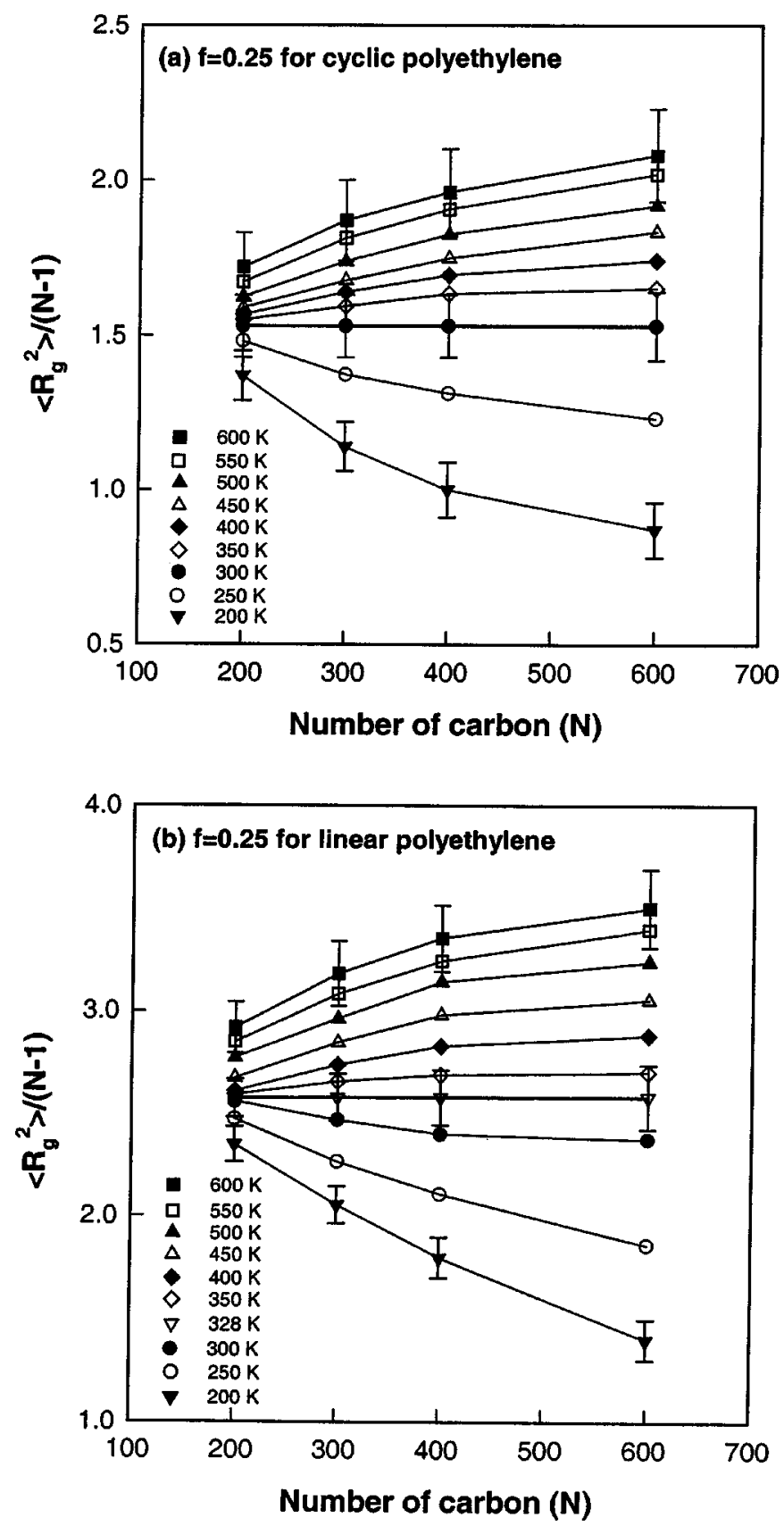

FIG. 5. Temperature dependence of the mean square radius of gyration normalized by number of bonds in a chain. This is for solvent scaling factor of 0.25 . This shows that the $\theta$ condition is at $300 \mathrm{~K}$ for c-PE and at $328 \mathrm{~K}$ for 1-PE. The error bars correspond to the rms deviation calculated by considering the 20-ns-long trajectory as 10 uncorrelated 2-ns-long segments. 


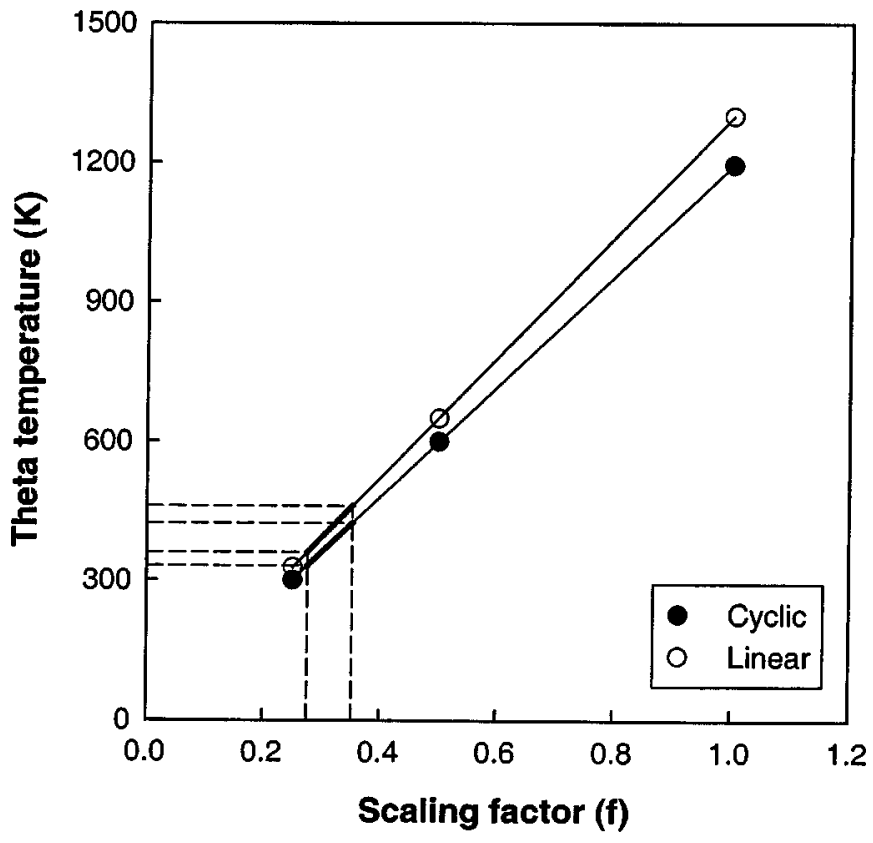

FIG. 6. Dependence of the calculated theta temperature on the solvent scale factor $f$. Common solvents lead to $f=0.28-0.35$, which are indicated by the vertical lines.

Figure 5 shows the predicted value of $\left\langle R_{g}^{2}\right\rangle /(N-1)$ as a function of chain length for various temperatures for a solvent scaling factor of $f=0.25$. We see that for $T=300 \mathrm{~K}$ this ratio is independent of chain length, establishing that $\theta=300$ $\mathrm{K}$ for cyclic polyethylene with $f=0.25$ solvent, in agreement with the results in Fig. 2(a). Similarly, Fig. 5 leads to $\theta=328$ $\mathrm{K}$ for linear PE in agreement with Fig. 2(b).

Figure 6 shows that the predicted theta temperature changes linearly with the solvent scaling factor for both cyclic and linear polyethylene, as shown in the following:

$$
\begin{array}{ll}
T_{\theta, \text { cyclic }}=1192.86 f+2.5 & \text { for cyclic polyethylene, } \\
T_{\theta, \text { linear }}=1296.57 f+3.0 & \text { for linear polyethylene }
\end{array}
$$

(least-squares correlation coefficient of 0.999).

The most used solvents have experimental theta temperatures for linear polyethylene between 360 and $460 \mathrm{~K} .^{89,90}$ The scale factor corresponding to this range of solvents is $f=0.28-0.35,{ }^{89,90}$ which is indicated by the vertical lines in Fig. 6. In this range of solvents (theta temperatures between 331 and $423 \mathrm{~K}$ ), we predict that the theta temperature of cyclic polyethylene is lower than that of linear polyethylene by $29-37 \mathrm{~K}$ or about $10 \%$.

\section{Characteristic ratios}

The characteristic ratio is defined only at the theta condition as shown in the following:

$$
\begin{aligned}
C_{\infty, \text { cyclic }}= & \lim _{N \rightarrow \infty} C_{N, \text { cyclic }} \\
& =\lim _{N \rightarrow \infty} \frac{12\left\langle R_{g}^{2}\right\rangle_{0, \text { cyclic }}}{N b^{2}} \text { for cyclic chain, }
\end{aligned}
$$

$$
\begin{aligned}
C_{\infty, \text { linear }} & =\lim _{N \rightarrow \infty} C_{N, \text { linear }} \\
& =\lim _{N \rightarrow \infty} \frac{6\left\langle R_{g}^{2}\right\rangle_{0, \text { linear }}}{N b^{2}} \text { for linear chain. }
\end{aligned}
$$

The value of the characteristic ratio indicates the effect of local interactions on chain conformation in the absence of excluded volume effects. Thus, for a freely jointed chain, this parameter has the value of 1.0. For convenience we discussed $C_{N}$ instead of $C_{\infty}$. The calculated characteristic ratios for various solvent scaling factors are shown in Table III. The value of characteristic ratio for linear polyethylene is in good agreement with experimental observations which ranges from ${ }^{89,90} 5.3$ to 7.1 with an average of $\sim 6.5$.

\section{Discussion}

Figure 4 shows that at each temperature the Flory exponent of cyclic polyethylene is larger than that of linear for all solvents $(f)$. This indicates that cyclic polyethylene experiences a greater excluded volume effect than does linear, as expected.

Figures 4 and 5 show that the theta temperature of cyclic polyethylene is $\sim 10 \%$ lower than that of linear polyethylene. Although an experimental $\theta$ for c-PE has not been reported, this depression of $\theta$ due to the cyclic architecture is consistent with experimental studies on polystyrene (PS), where cyclic PS has $\theta=28.0^{\circ} \mathrm{C}$, which is $2 \%$ lower than the $\theta=34.5^{\circ} \mathrm{C}$ for linear PS (at the same molecular weight), ${ }^{46,47,56}$ which was explained in terms of the topological constraint. ${ }^{27,28}$

Table III shows that the characteristic ratio decreases as the theta temperature increases. This implies that the unperturbed dimension of the polymer in a solvent with a higher theta temperature is smaller than that for a solvent with a lower theta temperature. This $\theta$ dependence of the characteristic ratio from this study is also observed experimentally. ${ }^{3,91,92}$ This result is interpreted as follows. At higher temperature, the population ratio of gauche to trans conformations decreases and polymer segments can easily overcome the conformational energy barrier between gauche and trans state. This can be thought of as a decrease in chain stiffness with increasing temperature. Figure 7 shows that the population in the trans state decreases as the theta temperature increases [higher scaling factor $(f)$ ] while the gauche state population increases. This indicates that the chain becomes more flexible at higher theta temperature and has smaller characteristic ratio.

We find that the characteristic ratio of cyclic polyethylene is $\sim 20 \%$ larger than that of linear PE. This is consistent with the dependence of the theta temperature on the characteristic ratio, since the theta temperature of cyclic polyethylene is lower than for linear PE.

The difference in characteristic ratio leads directly to a difference in the ratio of radius of gyration $\left(\left\langle R_{g}^{2}\right\rangle_{0, \text { cyclic }} /\right.$ $\left.\left\langle R_{g}^{2}\right\rangle_{0 \text {,inear }}\right)$ measured at $\theta$, as shown in Fig. 8. We find that this ratio varies from 0.59 to 0.57 for various solvent scaling factors $(f)$. This contrasts with the value of 0.5 from previous analytic theoretical prediction based on Eqs. (5a) and 

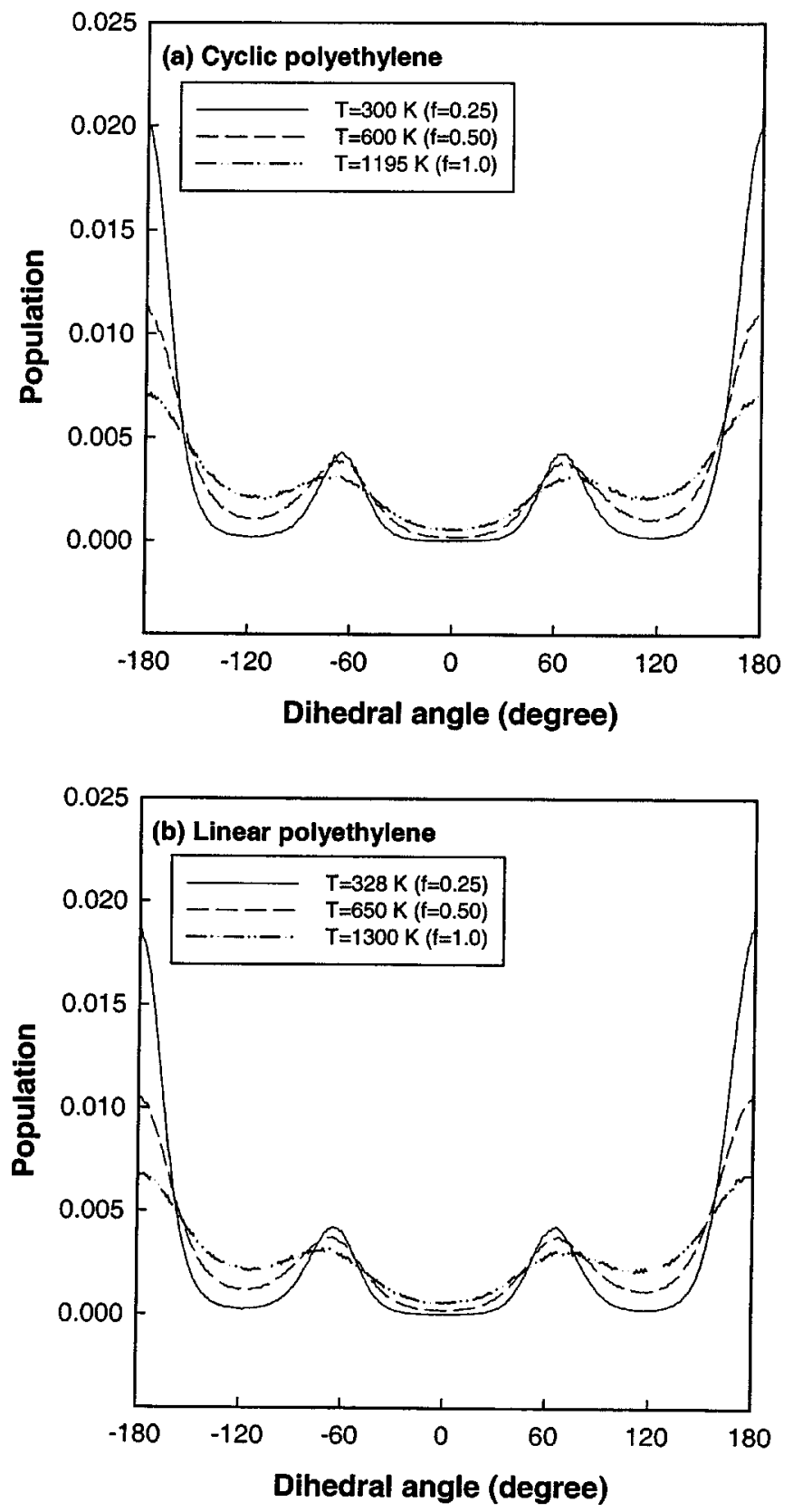

FIG. 7. Dihedral angle distribution at the theta temperatures for various solvent scaling factors $(f)$ : (a) cyclic PE, (b) linear PE.

(5b). Equations (5a) and (5b) can be rewritten by including the characteristic ratio at their individual theta temperatures:

$$
\begin{aligned}
& \left\langle R_{g}^{2}\right\rangle_{0, \text { cyclic }}=\frac{C_{N, \text { cyclic }}}{12}(N-1)\left\langle b^{2}\right\rangle \text { for cyclic chain, } \\
& \left\langle R_{g}^{2}\right\rangle_{0, \text { linear }}=\frac{C_{N, \text { linear }}}{6}(N-1)\left\langle b^{2}\right\rangle \text { for linear chain, } \\
& \left\langle R_{g}^{2}\right\rangle_{0, \text { cyclic }} /\left\langle R_{g}^{2}\right\rangle_{0, \text { linear }}=\frac{1}{2} \frac{C_{N, \text { cyclic }}}{C_{N, \text { linear }}} .
\end{aligned}
$$

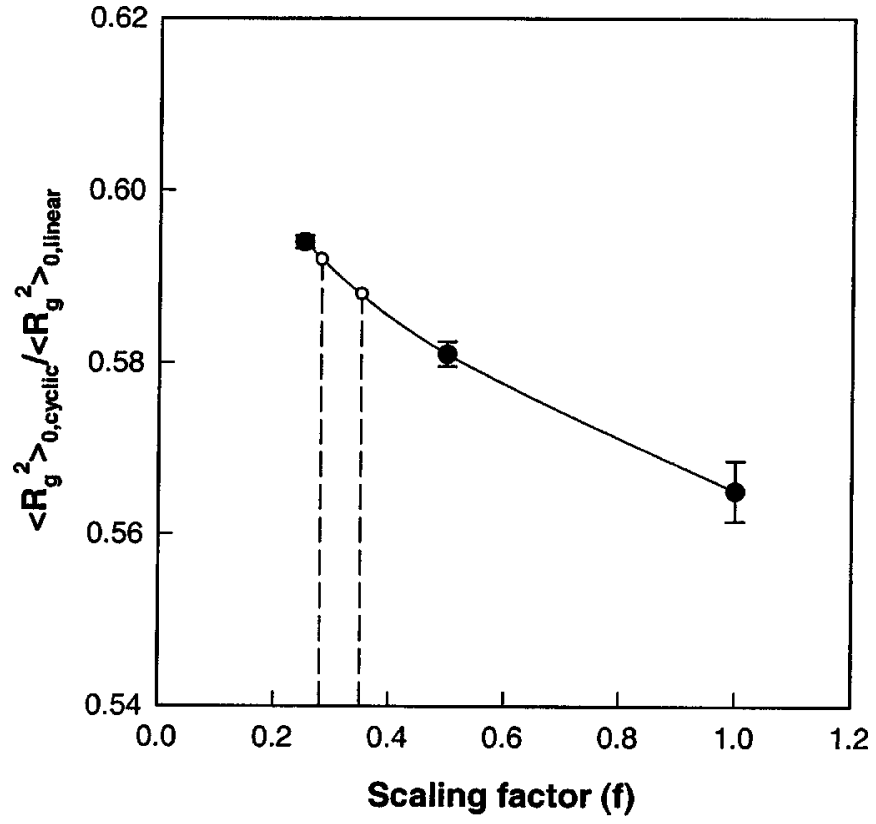

FIG. 8. Ratio of radius of gyration between cyclic and linear polyethylene at the $T=\theta$ as a function of solvent scaling factor. Common solvents lead to $f=0.28-0.35$, which are indicated by the vertical lines.

Thus, as the value of $C_{N \text {,cyclic }} / C_{N \text {, linear }}$ approaches 1.0 with increasing solvent scaling factor (poorer solvents), the value of $\left\langle R_{g}^{2}\right\rangle_{0, \text { cyclic }} /\left\langle R_{g}^{2}\right\rangle_{0 \text {,linear }}$ approaches the analytic theoretical prediction of 0.5 .

Earlier experimental studies on cyclic PS by Roover ${ }^{47}$ reported $\left\langle R_{g}^{2}\right\rangle_{0, \text { cyclic }} /\left\langle R_{g}^{2}\right\rangle_{0 \text {,inear }}=0.53$, which is in reasonable agreement with our results on PE.

In investigating the effect of a good solvent on chain conformations, Bloomfield and Zimm assumed that the nonGaussian nature of chain conformation is caused both by the excluded volume effect and by chain stiffness. ${ }^{20}$ This led to attempts to extend the concept of the characteristic ratio to describe the effect of local interactions on chain conformation in good solvents. Using

$$
\begin{aligned}
\left\langle R_{i j}^{2}\right\rangle_{\text {cyclic }}= & C_{N, \text { cyclic }}|i-j|^{1+\zeta}\left(N^{1+\zeta}-|i-j|^{1+\zeta}\right) / \\
& N^{1+\zeta}\left\langle b^{2}\right\rangle \text { for cyclic chain, } \\
\left\langle R_{i j}^{2}\right\rangle_{\text {linear }}= & C_{N, \text { linear }}|i-j|^{1+\zeta}\left\langle b^{2}\right\rangle \text { for linear chain, }
\end{aligned}
$$

where $\zeta=2 \nu-1$ and $\left\langle R_{i j}^{2}\right\rangle_{k}$ ( $k$ denotes cyclic or linear chain) is the mean square distance between segments $i$ and $j$, in the derivation of Eqs. (6a) and $(6 b)^{4,20}$ leads to

$$
\left\langle R_{g}^{2}\right\rangle_{\mathrm{cyclic}}=\frac{C_{N, \mathrm{cyclic}}}{2\left(6+5 \zeta+\zeta^{2}\right)}\left(1+\frac{\zeta}{3+2 \zeta}\right)(N-1)^{2 \nu}\left\langle b^{2}\right\rangle
$$

for cyclic chain,

$$
\left\langle R_{g}^{2}\right\rangle_{\text {linear }}=\frac{C_{N, \text { linear }}}{\left(6+5 \zeta+\zeta^{2}\right)}(N-1)^{2 \nu}\left\langle b^{2}\right\rangle
$$

for linear chain. 


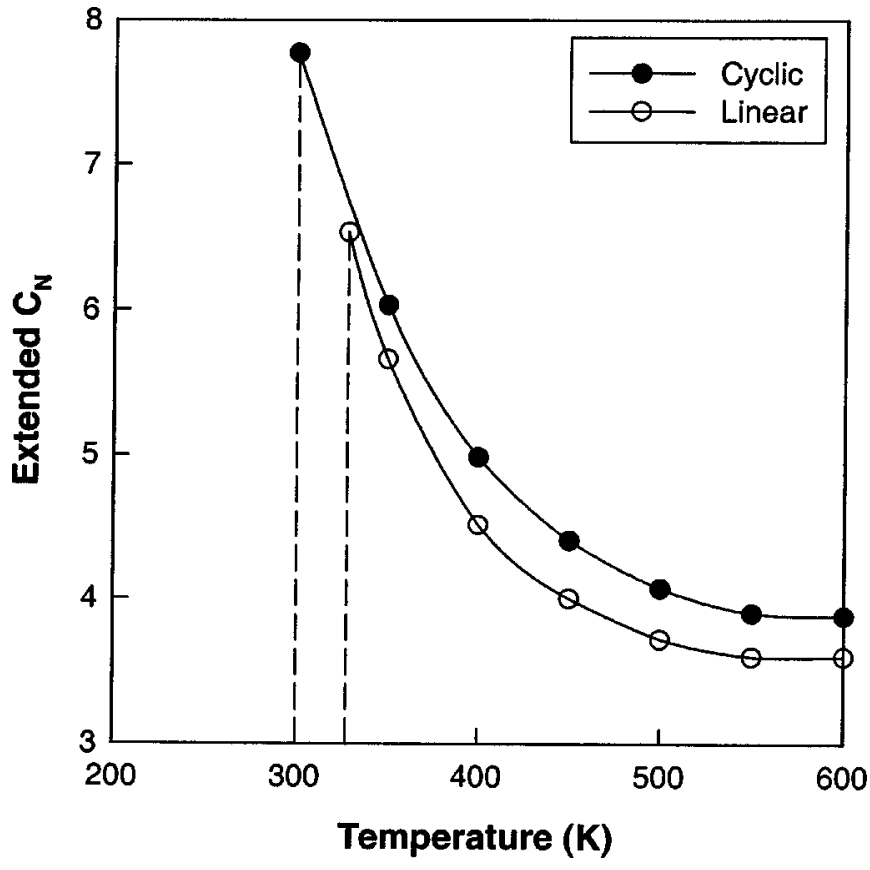

FIG. 9. Dependence of extended characteristic ratio on temperature for solvent scaling factor $f=0.25$. The $\theta$ temperature is indicated by vertical line for each use.

Figure 9 shows the extended characteristic ratio calculated for $f=0.25$ as a function of temperature. This temperature dependence was explained earlier in discussing Table III and Fig. 7, namely the chain is more flexible at higher temperature. Ignoring the decrease in characteristic ratio with increasing temperature would have led to a chain expansion in good solvents much larger than in our simulations. This indicates that ignoring such effects is an oversimplification.

The magnitude of the extended characteristic ratio for cyclic polyethylene is larger than that of linear counterpart at the same temperature, and at the same temperature the excluded volume effect for cyclic chain is greater than for linear chains (the cyclic chain architecture effect). Thus, the extended characteristic ratio is a function of both temperature and chain architecture.

Figure 10 shows $\left\langle R_{g}^{2}\right\rangle_{\text {cyclic }} /\left\langle R_{g}^{2}\right\rangle_{\text {linear }}$ as a function of the excluded volume parameter and temperature for $N=600$ and a solvent with $f=0.25$. Whereas analytical theories lead to $^{4,20,21}\left\langle R_{g}^{2}\right\rangle_{\text {cyclic }} /\left\langle R_{g}^{2}\right\rangle_{\text {linear }}$ increasing from 0.50 to 0.53 as the excluded volume parameter $(\zeta)$ is increased from 0.0 to 0.20 , our simulations lead to the opposite trend: $\left\langle R_{g}^{2}\right\rangle_{\text {cyclic }} /\left\langle R_{g}^{2}\right\rangle_{\text {linear }}$ decreases from 0.59 to 0.48 as shown in Fig. 10(a). This deviation from analytic theory arises because we find that

$$
\left\langle R_{g}^{2}\right\rangle_{\text {cyclic }} /\left\langle R_{g}^{2}\right\rangle_{\text {linear }}=\frac{1}{2}\left(1+\frac{\zeta}{3+2 \zeta}\right) \frac{C_{N, \text { cyclic }}}{C_{N, \text { linear }}},
$$

which also depends on $C_{N, \text { cyclic }} / C_{N \text {,linear }}$.

In Eq. (19), two competitive factors affect the behavior of $\left\langle R_{g}^{2}\right\rangle_{\text {cyclic }} /\left\langle R_{g}^{2}\right\rangle_{\text {linear }}$ : excluded volume effects and the chain stiffness. The results in Fig. 10(a) show that the contribution of excluded volume effect to $\left\langle R_{g}^{2}\right\rangle_{\text {cyclic }} /\left\langle R_{g}^{2}\right\rangle_{\text {linear }}$ is surpassed by the opposite contribution of chain stiffness.
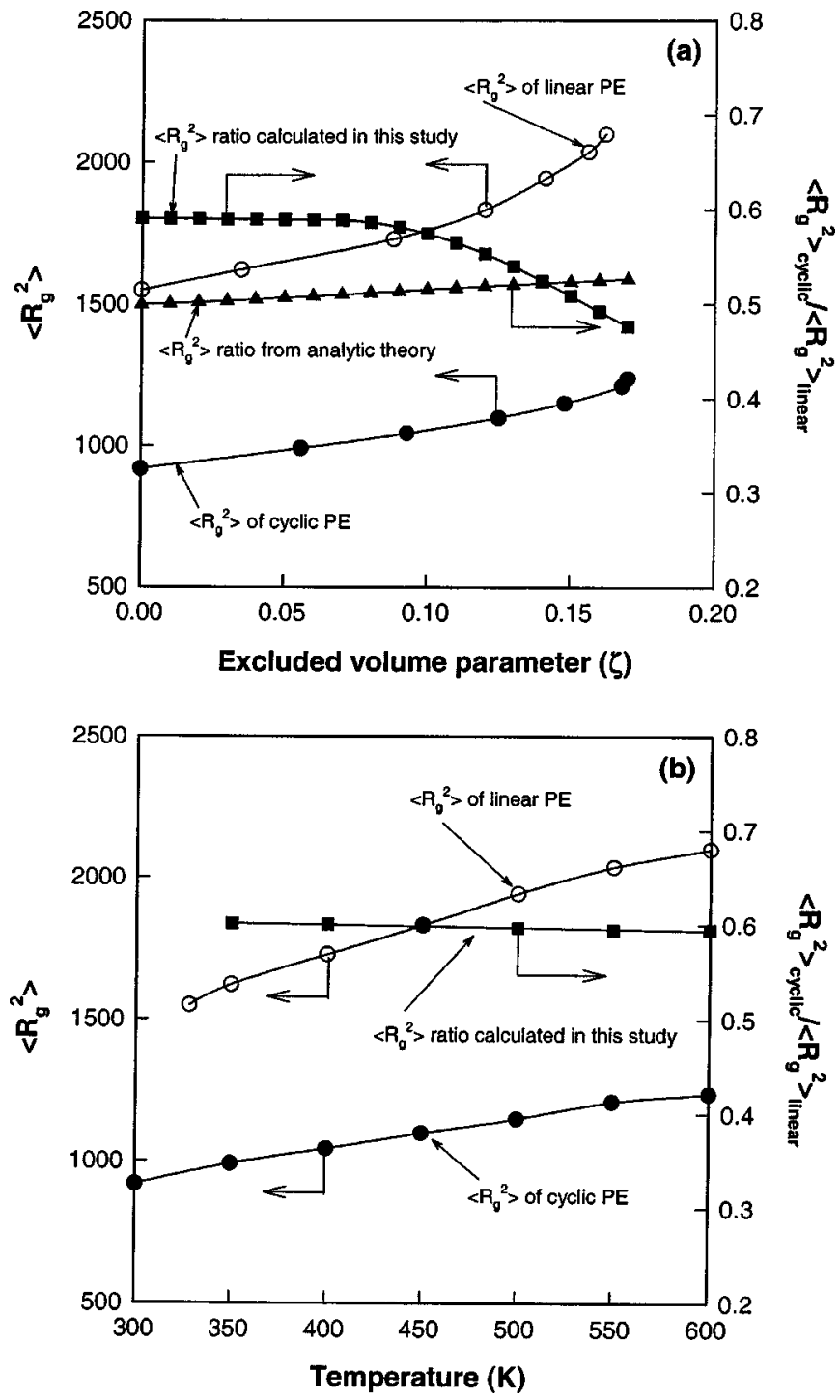

FIG. 10. (a) Dependence of radius of gyration on excluded volume parameter $(\xi)$; (b) dependence of radius on gyration on temperature. Both cases are for solvent scaling factors of $f=0.25$. Left axis: $\left\langle R_{g}^{2}\right\rangle$. Right axis: ratio between $\left\langle R_{g}^{2}\right\rangle$ for cyclic and linear chains.

Consequently, the chain expansion of cyclic polyethylene compared to linear polyethylene is smaller than predicted from analytic theory for the same solvent condition.

Figure 10(a) compares cyclic and linear polyethylene for various amounts of the excluded volume effect (at a given solvent condition). However, for comparison to experiment, it is much more useful to consider systems at the same temperature. As discussed in Figs. 3 and 5, the excluded volume effect felt by a cyclic chain is larger than for linear chain in the same solvent at the same temperature. Thus, to compare properties at the same temperature we must consider different solvent conditions with different excluded volume effects.

Figure 10(b) shows that the decrease of $\left\langle R_{g}^{2}\right\rangle_{\text {cyclic }} /$ $\left\langle R_{g}^{2}\right\rangle_{\text {linear }}$ is negligibly small (from 0.60 to 0.59 ) as temperature is increased from 350 to $600 \mathrm{~K}$. The decreasing rate of $\left\langle R_{g}^{2}\right\rangle_{\text {cyclic }} /\left\langle R_{g}^{2}\right\rangle_{\text {linear }}$ is smaller than that shown in Fig. 10(a) because the comparison at the same temperature means that 
cyclic polyethylene has larger excluded volume effect than linear counterpart.

Remarkably, Lutz et al. ${ }^{56}$ reported that $\left\langle R_{g}^{2}\right\rangle_{\text {cyclic }}$ / $\left\langle R_{g}^{2}\right\rangle_{\text {linear }}=0.53$ for PS in a good solvent, which is the same as Roover ${ }^{47}$ reported for the theta temperature for the same polymer using the same solvent. Thus, previous experimental comparisons between cyclic and linear polymer can be explained consistently by considering the relationship between temperature and solvent condition plus the distinct behavior of characteristic ratio according to the chain architecture.

\section{HYDRODYNAMIC PROPERTIES}

\section{A. The simplified Oseen tensor}

To characterize the effect of chain architecture on hydrodynamic properties, we calculated the intrinsic viscosity by combining our MD results with the modified analytic theory in which chain stiffness is described by the characteristic ratio. The hydrodynamic interaction between $i$ th and $j$ th segment in a polymer chain far apart from each other by distance $R_{i j}$ in a fluid is obtained by Oseen tensor $\left(\mathbf{T}_{i j}\right)$ in Eq. (20) which was simplified by Kirkwood and Riseman as shown in Eq. (21), ${ }^{65}$

$$
\begin{aligned}
& \mathbf{T}_{i j}=\frac{1}{8 \pi \eta_{0} R_{i j}}\left(\mathbf{I}+\frac{\mathbf{R}_{i j} \mathbf{R}_{i j}}{R_{i j}^{2}}\right), \\
& \left\langle\mathbf{T}_{i j}\right\rangle=\frac{1}{6 \pi \eta_{0}}\left\langle\frac{1}{\mathbf{R}_{i j}}\right),
\end{aligned}
$$

where $\mathbf{I}$ is a unit tensor and $\eta_{0}$ is solvent viscosity. In this approximation, the hydrodynamic interaction between every pair of polymer segments is treated as an averaged quantity according to the statistical conformations of polymer chain, $\left\langle 1 / \mathbf{R}_{i j}\right\rangle$. Thus, instead of $\left\langle 1 / \mathbf{R}_{i j}\right\rangle$ calculated from freely jointed chain in Eqs. (22a) and (22b) for cyclic and linear chain, respectively, ${ }^{4,20,21}$

$$
\begin{aligned}
\left\langle\mathbf{T}_{i j}\right\rangle & =\frac{1}{6 \pi \eta_{0}}\left\langle\frac{1}{\mathbf{R}_{i j}}\right\rangle \\
& =\frac{1}{\left(6 \pi^{3}\right)^{1 / 2}}\left[b^{2}|j-i|^{1+\zeta}\left(N^{1+\zeta}-|j-i|^{1+\zeta}\right) / N^{1+\zeta}\right]^{-1 / 2}
\end{aligned}
$$

for cyclic chain,

$\left\langle\mathbf{T}_{i j}\right\rangle=\frac{1}{6 \pi \eta_{0}}\left\langle\frac{1}{\mathbf{R}_{i j}}\right\rangle=\frac{1}{\left(6 \pi^{3}\right)^{1 / 2}}\left[b^{2}|j-i|^{1+\zeta}\right]^{-1 / 2}$

for linear chain,

we introduced explicitly the characteristic ratio into $\left\langle 1 / \mathbf{R}_{i j}\right\rangle$ as shown in the following:

$$
\begin{aligned}
\left\langle\mathbf{T}_{i j}\right\rangle= & \frac{1}{6 \pi \eta_{0}}\left\langle\frac{1}{\mathbf{R}_{i j}}\right\rangle \\
= & \frac{1}{\left(6 \pi^{3}\right)^{1 / 2}}\left[C _ { \mathrm { cyclic } } b ^ { 2 } | j - i | ^ { 1 + \zeta } \left(N^{1+\zeta}-\mid j\right.\right. \\
& \left.\left.-\left.i\right|^{1+\zeta}\right) / N^{1+\zeta}\right]^{-1 / 2} \quad \text { for cyclic chain, }
\end{aligned}
$$

$$
\begin{aligned}
\left\langle\mathbf{T}_{i j}\right\rangle & =\frac{1}{6 \pi \eta_{0}} \\
& =\left\langle\frac{1}{\mathbf{R}_{i j}}\right\rangle \frac{1}{\left(6 \pi^{3}\right)^{1 / 2}}\left[C_{\text {linear }} b^{2}|j-i|^{1+\zeta}\right]^{-1 / 2}
\end{aligned}
$$

for linear chain.

\section{B. The viscosity}

The equations in Sec. IV A lead to analytic equations for intrinsic viscosity of both cases in the following:

$$
\begin{aligned}
{[\eta]_{\text {cyclic }}=} & \frac{\left(12 \pi^{3}\right)^{1 / 2} N_{A}}{M} 2^{-\zeta / 2} N^{(1+\zeta) / 2} b\left\langle R_{g}^{2}\right\rangle_{\text {cyclic }} \\
& \times \sum_{j} \frac{2}{\lambda_{j}^{\prime}} \quad \text { for cyclic chain, } \\
{[\eta]_{\text {linear }}=} & \frac{\left(12 \pi^{3}\right)^{1 / 2} N_{A}}{M} 2^{-\zeta / 2} N^{(1+\zeta) / 2} b\left\langle R_{g}^{2}\right\rangle_{\text {linear }} \\
& \times \sum_{j} \frac{1}{\lambda_{j}^{\prime}} \quad \text { for linear chain. }
\end{aligned}
$$

This becomes

$$
\begin{aligned}
{[\eta]_{\text {cyclic }}=} & \frac{2\left(12 \pi^{3 i}\right)^{1 / 2} N_{A}}{24 M} 2^{-\zeta / 2} N^{(1-\zeta) / 2} C_{\text {cyclic }}^{-1 / 2} \frac{6+5 \zeta+\zeta^{2}}{\left(1+\frac{\zeta}{3+2 \zeta}\right)} \\
& \times b\left\langle R_{g}^{2}\right\rangle_{\text {cyclic }} \sum_{j} \frac{2}{\lambda_{j}^{\prime}} \quad \text { for cyclic chain, } \\
{[\eta]_{\text {linear }}=} & \frac{\left(12 \pi^{3}\right)^{1 / 2} N_{A}}{24 M} 2^{-\zeta / 2} N^{(1-\zeta / 2} C_{\text {linear }}^{-1 / 2}\left(6+5 \zeta+\zeta^{2}\right) \\
& \times b\left\langle R_{g}^{2}\right\rangle_{\text {linear }} \sum_{j} \frac{1}{\lambda_{j}^{\prime}} \quad \text { for linear chain, }
\end{aligned}
$$

where $N_{A}$ is Avogadro's number, $M$ is molecular weight of the polymer, and $\lambda_{j}^{\prime}$ is an eigenvalue from diagonalizing the position-to-velocity transformation matrix in the analytic theory ${ }^{4,20,93}$ which is given in the following: ${ }^{62,64}$

$$
\begin{gathered}
\text { for linear chain, } \\
C_{\delta}=(2 \pi)^{-1 / 2} \int_{0}^{x} t^{-\delta} \cos t d t, \\
S_{\delta}(2 \pi)^{-1 / 2} \int_{0}^{x} t^{-\delta} \sin t d t,
\end{gathered}
$$$$
\lambda_{j}^{\prime}=2^{1-\delta} \pi^{2} j^{2} \int_{0}^{1}\left(\frac{x}{2}\right)^{-\delta}\left[1-\left(\frac{x}{2}\right)^{2 \delta}\right]^{-1 / 2}
$$$$
\times \cos (\pi j x) d x \text { for cyclic chain, }
$$$$
\lambda_{j}^{\prime}=(2 \pi)^{1 / 2} 2^{-\delta}(\pi j)^{\delta}\left[\pi j C_{\delta}(\pi j)-\delta S_{\delta}(\pi j)\right]
$$

where $\delta=(1+\zeta) / 2$.

In order to calculate the value of intrinsic viscosity, the excluded volume parameters were determined from the Flory exponents shown in Table II, and the infinite sum of $1 / \lambda_{j}^{\prime}$ 
TABLE IV. The excluded volume parameters and the eigenvalues calculated for cyclic and linear polyethylene. See Eqs. (26) and (27).

\begin{tabular}{lccccccc}
\hline \hline \multicolumn{3}{c}{ Cyclic polyethylene } & & \multicolumn{3}{c}{ Linear polyethylene } \\
\cline { 1 - 3 } \cline { 6 - 8 }$T(\mathrm{~K})$ & $\zeta$ & $\Sigma_{j}^{\infty} \frac{2}{\lambda_{j}^{\prime}}$ & & $T(\mathrm{~K})$ & $\zeta$ & $\Sigma_{j}^{\infty} \frac{1}{\lambda_{j}^{\prime}}$ \\
\hline 300 & 0.000 & 0.3833 & & 328 & 0.000 & 0.5823 \\
350 & 0.056 & 0.3210 & & 350 & 0.035 & 0.5294 \\
400 & 0.093 & 0.2838 & & 400 & 0.088 & 0.4584 \\
450 & 0.125 & 0.2590 & & 450 & 0.120 & 0.4206 \\
500 & 0.148 & 0.2412 & & 500 & 0.141 & 0.3970 \\
550 & 0.168 & 0.2271 & & 550 & 0.156 & 0.3809 \\
600 & 0.170 & 0.2245 & & 600 & 0.162 & 0.3747 \\
\hline \hline
\end{tabular}

was evaluated numerically using MATHEMATICA of Wolfram Research, Inc. ${ }^{94}$ as summarized in Table IV. This leads to values that are in good agreement with the values in Bloomfield-Zimm. ${ }^{20}$
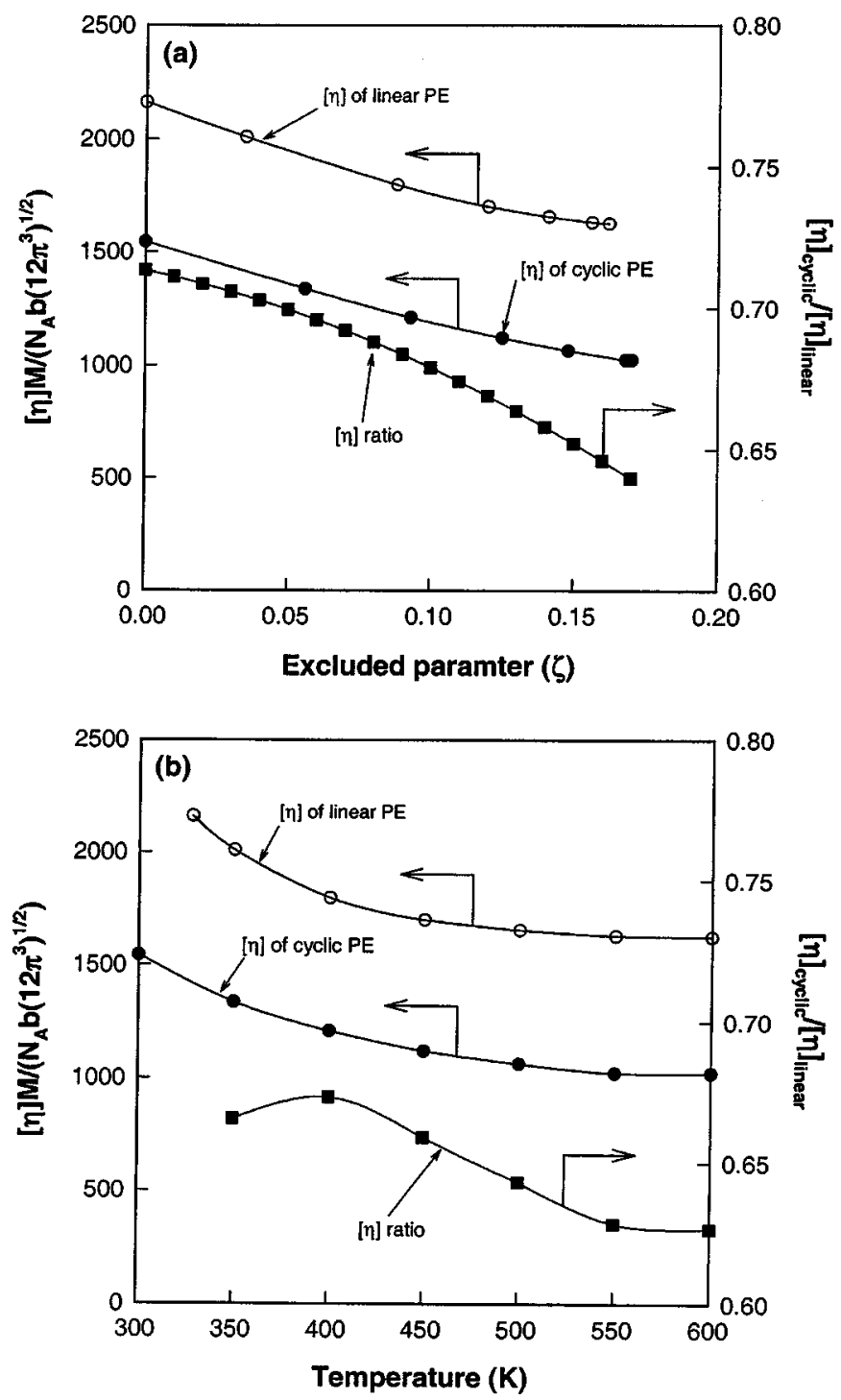

FIG. 11. (a) Intrinsic viscosity as a function of excluded volume parameter $(\xi)$; (b) intrinsic viscosity as a function of temperature. Both cases are for solvent scaling factors of $f=0.25$. Left axis: intrinsic viscosity. Right axis: intrinsic viscosity ratio.
Figure 11(a) shows that the intrinsic viscosity decreases with increasing excluded volume parameter from the theta condition $(\zeta=0)$ to the good solvent condition $(\zeta=0.17)$, and simultaneously, the value of $[\eta]_{\text {cyclic }} /[\eta]_{\text {linear }}$ monotonically decreases from 0.71 to 0.64 , in good agreement with analytic theoretical predictions. ${ }^{4}$ The value of $[\eta]_{\text {cyclic }} /[\eta]_{\text {linear }}$ at the theta condition, 0.71 , is slightly larger than the prediction from analytic theory, 0.66 . This is probably caused by the larger value of $\left\langle R_{g}^{2}\right\rangle_{\text {cyclic }} /\left\langle R_{g}^{2}\right\rangle_{\text {linear }}=0.59$, compared to the analytic theory value, 0.5 . We believe that this can rationalize the experimental observations ${ }^{95,96}$ in which intrinsic viscosity ratio of polystyrene decreases from 0.71 to 0.62 along with the solvent condition. On the other hand, the ratio of viscosity at the same temperature need not exhibit such simple behavior in Fig. 11(b). This is seen in the different shape of the curves for the intrinsic viscosity around $T$ $=350 \mathrm{~K}$, which is probably due to the difference in the decreasing rate of the intrinsic viscosity with temperature. Indeed, the comparison between the experimental intrinsic viscosity of cyclic and linear polymers for PS (leading to the value of 0.66 ) was performed at the theta temperature of linear polymer, not at the individual theta temperature of each case. $^{47,56}$

These results are not consistent with the analytic theoretical predictions, which are valid when the comparison is at the same excluded-volume-free condition. These analytic theory results seem questionable because the cyclic chain experiences more excluded volume effect at the theta condition of linear chain and its characteristic ratio is equal to or larger than that of linear one. Therefore, systematic experimental studies would be useful to confirm the predictions of the analytic theory and to test the predictions from our simulation.

\section{SUMMARY}

The dilute solution properties of cyclic polyethylene were investigated by combining canonical (Nose-Hoover) simulations with modified analytic theory.

To predict the temperature dependence of solvent effects, we introduce a solvent scaling factor $(f)$ into the van der Waals interaction. The solvent scaling factor is related to the theta temperature, which allows the scaling factor to be estimated for experimental solvents.

We find that the theta temperature for cyclic PE is $\sim 10 \%$ lower than that of linear PE.

To describe the effects of solvent and chain stiffness over a range of temperatures, we extended the concept of the characteristic ratio (originally defined only at the theta temperature). We find that the extended characteristic ratio decreases as the temperature is increased, indicating that the chain becomes more flexible with increasing temperature. This allows us to characterize the effect of temperature and solvent on the size of the polymer chains. We find that the extended characteristic ratio of cyclic polyethylene is $6 \%-$ $13 \%$ larger than linear PE (at the same temperature) for solvent conditions corresponding to $n$-pentane. This implies that the extended characteristic ratio is influenced by the chain architecture as well as by temperature. 
We find that $\left\langle R_{g}^{2}\right\rangle_{\text {cyclic }} /\left\langle R_{g}^{2}\right\rangle_{\text {linear }}$ decreases from 0.59 to 0.48 as the excluded volume parameter $(\zeta)$ is increased, which is opposite the result of analytical theory based on the freely jointed chain model. We interpret this to indicate that the contribution of excluded volume effect to $\left\langle R_{g}^{2}\right\rangle_{\text {cyclic }} /\left\langle R_{g}^{2}\right\rangle_{\text {linear }}$ is surpassed by the competitive contribution of chain stiffness (expressed by the characteristic ratio).

We find that for $n$-pentane solvent $\left\langle R_{g}^{2}\right\rangle_{\text {cyclic }} /\left\langle R_{g}^{2}\right\rangle_{\text {linear }}$ $=0.59$ is independent of temperature. This is consistent with experimental observations for polystyrene, where the ratio is 0.53 .

To calculate the intrinsic viscosity for cyclic and linear polyethylene, we combined the conformational statistics from MD simulations with the Bloomfield-Zimm type theory, introducing the extended characteristic ratio. We find that the value of $[\eta]_{\text {cyclic }} /[\eta]_{\text {linear }}$ decreases from 0.71 to 0.64 as a function of excluded volume parameter $(\zeta)$. This value is $7 \%$ larger than from analytic theory.

\section{ACKNOWLEDGMENTS}

We particularly thank Professor Robert Grubbs for alerting us to his results prior to publication and to Diego Benitez and Professor Grubbs for spirited discussions. This research was partially supported by NSF (CHE 99-85574) and ARO (DAAG55-97-1-0126). The computational facilities were provided by DURIP grants from ARO and ONR, and MRI grant from NSF, and a SUR grant from IBM. The facilities of the Materials and Process Simulation Center are also supported by DOE (ASCI ASAP), NSF (CHE), MURI-ARO, MURI-ONR, General Motors, Chevron Texaco, SeikoEpson, Beckman Institute, and Asahi Kasei.

${ }^{1}$ C. W. Bielawski, D. Benitez, and R. H. Grubbs, Science 297, 2041 (2002). ${ }^{2}$ P. J. Flory, Principles of Polymer Chemistry (Cornell University Press, Ithaca, NY, 1953).

${ }^{3}$ P. J. Flory, Statistical Mechanics of Chain Molecules (Wiley, New York, 1969).

${ }^{4}$ H. Yamakawa, Modern Theory of Polymer Solutions (Harper \& Row, New York, 1971).

${ }^{5}$ K. F. Freed, Renormalization Group Theory of Macromolecules (Wiley, New York, 1987).

${ }^{6}$ H. Fujita, Polymer Solutions (Elsevier, New York, 1990).

${ }^{7}$ J. D. Cloizeaux and G. Jannink, Polymers in Solution: Their Modeling and Structure (Oxford University Press, Oxford, 1990).

${ }^{8}$ H. A. Kramers, Physica (Amsterdam) 11, 1 (1944).

${ }^{9}$ H. A. Kramers, J. Chem. Phys. 14, 415 (1946).

${ }^{10}$ B. H. Zimm and W. H. Stockmayer, J. Chem. Phys. 17, 1301 (1949).

${ }^{11}$ W. Fiers and R. L. Sinsheimer, J. Mol. Biol. 5, 408 (1962).

${ }^{12}$ A. D. Hershey, E. Burgi, and L. Ingraham, Proc. Natl. Acad. Sci. U.S.A. 49, 748 (1963).

${ }^{13}$ R. Dulbecco and M. Vogt, Proc. Natl. Acad. Sci. U.S.A. 50, 236 (1963).

${ }^{14}$ R. Weil and J. Vinograd, Proc. Natl. Acad. Sci. U.S.A. 50, 730 (1963).

${ }^{15}$ A. K. Kleinschmidt, A. Burton, and R. L. Sinsheimer, Science 142, 961 (1963).

${ }^{16}$ A. Burton and R. L. Sinsheimer, Science 142, 962 (1963).

${ }^{17}$ B. Chandler, M. Hayashi, M. N. Hayashi, and S. Spiegelman, Science 143, 47 (1964).

${ }^{18}$ C. A. Thomas and L. A. MacHattie, Proc. Natl. Acad. Sci. U.S.A. 52, 1297 (1964).

${ }^{19}$ E. F. Casassa, J. Polym. Sci., Part A: Gen. Pap. 3, 605 (1965).

${ }^{20}$ V. Bloomfield and B. H. Zimm, J. Chem. Phys. 44, 315 (1966).

${ }^{21}$ M. Fukatsu and M. Kurata, J. Chem. Phys. 44, 4539 (1966).

${ }^{22}$ H. B. Gray, V. A. Bloomfield, and J. E. Hearst, J. Chem. Phys. 46, 1493 (1967).

${ }^{23}$ S. Imai, J. Chem. Phys. 51, 1732 (1969).
${ }^{24}$ J. Naghizadeh and H. Sotobayashi, J. Chem. Phys. 60, 3104 (1974).

${ }^{25}$ W. Bruns and J. Naghizadeh, J. Chem. Phys. 65, 747 (1976).

${ }^{26}$ W. Burchard and M. Schmidt, Polymer 21, 745 (1980).

${ }^{27}$ K. Iwata and T. Kimura, J. Chem. Phys. 74, 2039 (1981).

${ }^{28}$ K. Iwata, Macromolecules 22, 3702 (1989).

${ }^{29}$ J. J. Prentis, J. Chem. Phys. 76, 1574 (1982).

${ }^{30}$ Y.-D. Chen, J. Chem. Phys. 78, 5191 (1983).

${ }^{31}$ J. M. Wiest, S. R. Burdette, T. W. Liu, and R. B. Bird, J. Non-Newtonian Fluid Mech. 24, 279 (1987).

${ }^{32}$ T. W. Liu and H. C. Oettinger, J. Chem. Phys. 87, 3131 (1987).

${ }^{33}$ M. Bishop and C. J. Seltiel, J. Chem. Phys. 89, 1159 (1988).

${ }^{34}$ J. M. G. Bernal, M. M. Tirado, J. J. Freire, and J. G. d. 1. Torre, Macromolecules 23, 3357 (1990).

${ }^{35}$ K. Koniaris and M. Muthukumar, J. Chem. Phys. 95, 2873 (1991).

${ }^{36}$ K. Koniaris and M. Muthukumar, Phys. Rev. Lett. 66, 2211 (1991).

${ }^{37}$ G. Merkle, W. Burchard, P. Lutz, K. F. Freed, and J. Gao, Macromolecules 26, 2736 (1993).

${ }^{38}$ J. K. Johnson, J. Chem. Phys. 104, 1729 (1996).

${ }^{39}$ W. Burchard, E. Michel, and V. Trappe, Macromolecules 29, 5934 (1996).

${ }^{40}$ S. Brown and G. Szamel, J. Chem. Phys. 109, 6184 (1998).

${ }^{41}$ M. Muller, J. P. Wittmer, and M. E. Cates, Phys. Rev. E 61, 4078 (2000).

${ }^{42}$ G. Zifferer and W. Preusser, Macromol. Theory Simul. 10, 397 (2001).

${ }^{43}$ E. G. Timoshenko, Y. A. Kuznetsov, and R. Connolly, J. Chem. Phys. 116, 3905 (2002).

${ }^{44}$ P. Calabrese, A. Pelisetto, and E. Vicari, J. Chem. Phys. 116, 8191 (2002).

${ }^{45}$ A. M. Rubio, J. J. Freire, M. Bishop, and J. H. R. Clarke, Macromolecules 28, 2240 (1995).

${ }^{46}$ J. Roovers and P. M. Toporowski, Macromolecules 16, 843 (1983).

${ }^{47}$ J. Roovers, J. Polym. Sci., Polym. Phys. Ed. 23, 1117 (1985).

${ }^{48}$ J. Roovers, Macromolecules 18, 1359 (1985).

${ }^{49}$ K. Dodgson and J. A. Semylen, Polymer 18, 1265 (1977).

${ }^{50}$ K. Dodgson, D. Sympson, and J. A. Semlyen, Polymer 19, 1285 (1978).

${ }^{51}$ J. S. Higgins, K. Dodgson, and J. A. Semlyen, Polymer 20, 553 (1979).

${ }^{52}$ J. S. Higgins, K. Ma, L. K. Nicholson, J. B. Hayter, K. Dodgson, and J. A. Semyen, Polymer 24, 793 (1983).

${ }^{53}$ C. J. C. Edwards, R. W. Richards, R. F. T. Stepto, K. Dodgson, J. S. Higgins, and J. A. Semlyen, Polymer 25, 365 (1984).

${ }^{54}$ S. J. Clarson, J. A. Semlyen, J. Horska, and R. F. T. Stepto, Polym. Commun. 27, 31 (1986).

${ }^{55}$ M. Duval, P. Luts, and C. Strazielle, Makromol. Chem., Rapid Commun. 6, 71 (1985).

${ }^{56}$ P. Lutz, G. B. McKenna, P. Rempp, and C. Strazielle, Makromol. Chem., Rapid Commun. 7, 599 (1986).

${ }^{57}$ G. Hadziioannou, P. M. Cotts, G. T. Brinke, C. C. Han, P. Luts, C. Strazielle, P. Rempp, and A. J. Kovacs, Macromolecules 20, 493 (1987).

${ }^{58}$ M. Ragnetti, D. Geiser, H. Hoeker, and R. C. Oberthuer, Makromol. Chem. 186, 1701 (1985).

${ }^{59}$ B. H. Zimm, J. Chem. Phys. 24, 269 (1956).

${ }^{60}$ J. P. E. Rouse, J. Chem. Phys. 21, 1272 (1953).

${ }^{61}$ A. Peterlin, J. Chem. Phys. 23, 2464 (1955).

${ }^{62}$ O. B. Ptitsyn and Y. E. Eisner, Zh. Fiz. Khim. 32, 2464 (1958).

${ }^{63}$ M. Kurata, H. Yamakawa, and E. Teramoto, J. Chem. Phys. 28, 785 (1958).

${ }^{64}$ N. W. Tschoegl, J. Chem. Phys. 40, 473 (1964).

${ }^{65}$ J. G. Kirkwood and J. Riseman, J. Chem. Phys. 16, 565 (1948).

${ }^{66}$ M. Fixman, J. Chem. Phys. 23, 1656 (1955).

${ }^{67}$ C. W. Pyun and M. Fixman, J. Chem. Phys. 42, 3838 (1965).

${ }^{68}$ C. W. Pyun and M. Fixman, J. Chem. Phys. 44, 2107 (1966).

${ }^{69}$ M. Fixman, Macromolecules 14, 1710 (1981).

${ }^{70}$ J. I. Siepmann, S. Karaborni, and B. Smit, Nature (London) 365, 330 (1993).

${ }^{71}$ B. Smit, S. Karaborni, and J. I. Siepmann, J. Chem. Phys. 102, 2126 (1995).

${ }^{72}$ M. G. Martin and J. I. Siepmann, J. Am. Chem. Soc. 119, 8921 (1997).

${ }^{73}$ J. P. Nicolas and B. Smit, Mol. Phys. 100, 2471 (2002).

${ }^{74}$ A. Sariban, J. Brickman, J. V. Ruiten, and R. J. Meier, Macromolecules 25, 5950 (1992).

${ }^{75}$ A. Sariban, T. Mosell, and J. Brickman, Macromol. Theory Simul. 3, 963 (1994).

${ }^{76}$ M. Destree, A. Lyulin, and J.-P. Ryckaert, Macromolecules 29, 1721 (1996).

${ }^{77}$ S. Nose, J. Chem. Phys. 81, 511 (1984).

${ }^{78}$ S. Nose, Mol. Phys. 52, 255 (1984).

${ }^{79}$ S. Nose, Mol. Phys. 57, 187 (1986). 
${ }^{80}$ W. G. Hoover, Phys. Rev. A 31, 1695 (1985).

${ }^{81}$ Accelyrs Inc., CERIUS2 Modeling Environment, Release 4.0 (Accelrys Inc., San Diego, 1999).

${ }^{82}$ K.-T. Lim, S. Brunett, M. Iotov, R. B. McClurg, N. Vaidehi, S. Dasgupta, S. Taylor, and W. A. Goddard, J. Comput. Chem. 18, 501 (1997).

${ }^{83}$ G. Gao, T. Cagin, and W. A. Goddard, Phys. Rev. Lett. 80, 5556 (1998).

${ }^{84}$ T. Cagin, G. Wang, R. Martin, N. Breen, and I. W. A. Goddard, Nanotechnology 11, 77 (2000).

${ }^{85}$ T. Cagin, G. Wang, R. Martin, G. Zamanakos, N. Vaidehi, D. T. Mainz, and W. A. Goddard, Comput. Theor. Polym. Sci. 11, 345 (2001).

${ }^{86}$ F. L. McCrackin, J. Mazur, and C. M. Guttman, Macromolecules 6, 859 (1973).

${ }^{87}$ J. G. Curro and D. W. Scharfer, Macromolecules 13, 1199 (1980).

${ }^{88}$ A. Milchev, W. Paul, and K. Binder, J. Chem. Phys. 99, 4786 (1993).

${ }^{89}$ Polymer Handbook, edited by J. Brandup and E. H. Immergut (Wiley, New York, 1989).
${ }^{90}$ Physical Properties of Polymer Handbook, edited by J. E. Mark (AIP, Woodbury, 1996).

${ }^{91}$ R. Chiang, J. Phys. Chem. 70, 2348 (1966).

${ }^{92}$ A. Nakajima, H. Hamada, and S. Hayashi, J. Polym. Sci., Part C: Polym. Symp. 15, 285 (1966).

${ }^{93}$ B. H. Zimm, G. M. Roe, and L. F. Epstein, J. Chem. Phys. 24, 279 (1956).

${ }^{94}$ Wolfram Research Inc., MATHEMATICA 4.0 (Wolfram_Research_Inc., Champaign, 2000).

${ }^{95}$ G. Hild, C. Strazielle, and P. Rempp, Eur. Polym. J. 19, 721 (1983).

${ }^{96}$ R. Qian and T. Cao, Makromol. Chem. 188, 1757 (1987).

${ }^{97}$ S. J. Weiner, P. A. Kollman, D. A. Case, U. C. Singh, C. Ghio, G. Alagona, S. Profeta, and P. Weiner, J. Am. Chem. Soc. 106, 765 (1984).

${ }^{98}$ S. J. Weiner, P. A. Kollman, D. T. Nguyen, and D. A. Case, J. Comput. Chem. 7, 230 (1986)

${ }^{99}$ W. L. Jorgensen, F. D. Madura, and C. J. Swenson, J. Am. Chem. Soc. 106, 6638 (1984) 